\title{
On continuity and compactness of some nonlinear operators in the spaces of functions of bounded variation
}

\author{
Dariusz Bugajewski ${ }^{1}$ • Jacek Gulgowski² ${ }^{2}$ \\ Piotr Kasprzak ${ }^{1}$
}

Received: 3 April 2015 / Accepted: 20 July 2015 / Published online: 21 August 2015

(C) The Author(s) 2015. This article is published with open access at Springerlink.com

\begin{abstract}
In this paper, we deal with one of the basic problems of the theory of autonomous superposition operators acting in the spaces of functions of bounded variation, namely the problem concerning their continuity. We basically consider autonomous superposition operators generated by analytic functions or functions of $C^{1}$-class. We also investigate the problem of compactness of some classical linear and nonlinear operators acting in the space of functions of bounded variation in the sense of Jordan. We apply our results to the examination of the existence and the topological properties of solutions to nonlinear equations in those spaces.
\end{abstract}

Keywords Acting condition - Aronszajn-type theorem · Autonomous (nonautonomous) superposition operator $\cdot$ Bernstein polynomials $\cdot$ Compact operator $\cdot$ Hammerstein integral equation - Linear integral operator - Locally bounded mapping - Modulus of continuity $\cdot p$ variation $\cdot$ Positive solution $\cdot R_{\delta}$-set $\cdot$ Variation in the sense of Jordan · Volterra-Hammerstein integral equation $\cdot \phi$-function $\cdot \phi$-variation

Mathematics Subject Classification Primary 47H30 - 26A45; Secondary 45G10 • 45D05

Piotr Kasprzak

kasp@amu.edu.pl

Dariusz Bugajewski

ddbb@amu.edu.pl

Jacek Gulgowski

dzak@mat.ug.edu.pl

1 Optimization and Control Theory Department, Faculty of Mathematics and Computer Science, Adam Mickiewicz University, ul. Umultowska 87, 61-614 Poznań, Poland

2 Institute of Mathematics, University of Gdańsk, ul. Wita Stwosza 57, 80-952 Gdańsk, Poland 


\section{Introduction}

In the recently published monograph [2], on p. 6 there are stated three basic problems concerning nonautonomous superposition operators acting in the space of functions of bounded variation in the sense of Jordan. The first problem concerns necessary and sufficient conditions which would guarantee that the nonautonomous superposition operator maps the space of functions of bounded variation in the sense of Jordan into itself and is locally bounded. In the paper [7] Bugajewska et al. have given the answer to this problem proving, in particular, the following

Theorem 1 Suppose that $f:[0,1] \times \mathbb{R} \rightarrow \mathbb{R}$ is a given function. The following conditions are equivalent:

1. the nonautonomous superposition operator $F$, generated by $f$, maps the space $B V[0,1]$ into itself and is locally bounded;

2. for every $r>0$, there exists a constant $M_{r}>0$ such that for every $k \in \mathbb{N}$, every finite partition $0=t_{0}<\cdots<t_{k}=1$ of the interval $[0,1]$ and every finite sequence $u_{0}, u_{1}, \ldots, u_{k} \in[-r, r]$ with $\sum_{i=1}^{k}\left|u_{i}-u_{i-1}\right| \leq r$, the following inequalities hold

$$
\sum_{i=1}^{k}\left|f\left(t_{i}, u_{i}\right)-f\left(t_{i-1}, u_{i}\right)\right| \leq M_{r} \quad \text { and } \quad \sum_{i=1}^{k}\left|f\left(t_{i-1}, u_{i}\right)-f\left(t_{i-1}, u_{i-1}\right)\right| \leq M_{r} .
$$

(In the above theorem, $B V[0,1]$ denotes the Banach space of all functions $x:[0,1] \rightarrow \mathbb{R}$ of bounded variation in the sense of Jordan endowed with the norm $\|x\|_{B V}=|x(0)|+\bigvee_{0}^{1} x$; for more details see Sect. 2.)

The third problem mentioned in the monograph [2] concerns the continuity of autonomous and nonautonomous superposition operators described above. In connection with this problem, we would like to draw the reader's attention to the paper by Morse [23]. In Remark 5, we provide more comments on Morse's results contained in that paper.

In the first part of this paper, we deal with the continuity of autonomous superposition operators acting in the space of functions of bounded variation in the sense of Jordan. In particular, we prove that if the generator of an autonomous superposition operator is analytic or of $C^{1}$-class, then that operator is continuous. Let us emphasize that our approach to the problem of continuity of autonomous superposition operator is much simpler than that proposed by Morse. It is based on some techniques developed by us in connection with the investigation of mappings of higher order in the spaces of functions of bounded variation in the sense of Jordan as well as on Bernstein polynomials.

In Chapter 7 of the monograph [2], the authors discuss some applications of functions of bounded variation and nonlinear superposition operators acting in such spaces of functions to the theory of nonlinear integral equations. These applications contain and develop the existence and uniqueness results from the paper [8]. Let us also add that the proofs of all those results are based on the Banach contraction principle.

In this paper, we are going to establish that the basic conditions considered in the paper [8] imply the compactness of the Hammerstein integral operator as well as the VolterraHammerstein integral operator. As a consequence of this fact, one can use the Schauder-type fixed-point theorems to prove the existence of solutions to nonlinear integral equations under consideration in the classes of functions of bounded variation in the sense of Jordan. In the case of nonlinear Volterra-Hammerstein integral equation, we are also able to describe the topological structure of continuous solution sets to that equation which are of bounded varia- 
tion in the sense of Jordan. According to our best knowledge, it is the first attempt to establish an Aronszajn-type result for such solutions.

Let us emphasize that the investigation of solutions to nonlinear integral equations in the spaces of functions of bounded variation seems to be interesting for at least a few reasons. First, solutions to many nonlinear equations which describe concrete physical phenomena are functions of bounded variation in the sense of Jordan. We refer the reader to the papers [5] and [18] in which the authors deal with bounded variation solutions to nonlinear Volterra integral equations which describe, in particular, models of behavior of population, where a probability of death depends on age.

Finally, let us mention that functions of bounded variation also possess essential applications, for example, in the geometric measure theory (see, e.g., [1,22]), in image processing, analysis and recovery (see, e.g., $[11,12,15,16,27]$ ) or in the theory of Fourier series (see [30]). Let us add that some of those applications are based on the usage of the Mumford-Shah functional.

\section{Preliminaries}

Notation By $\mathbb{N}$, we denote the set of positive integers. Moreover, throughout the paper by $I$, we will denote the unit interval $[0,1]$.

The closed ball in a normed space $X$ with center at $x$ and radius $r \in(0,+\infty)$ will be denoted by $B_{X}(x, r)$. For simplicity, instead of $B_{\mathbb{R}}(x, r)$, we will simply write $[x-r, x+r]$. As usual, by $L^{p}(J)$ we will denote the Banach space of all the equivalence classes of real-valued functions defined on a bounded interval $J \subseteq \mathbb{R}$ which are either Lebesgue integrable with $p$ th power, if $p \in[1,+\infty)$, or essentially bounded, if $p=+\infty$, endowed

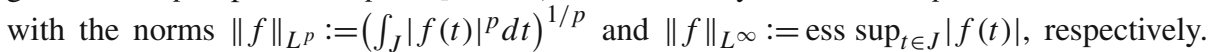
The Lebesgue measure will be denoted by $\mu$. By $B(I)$, we will denote the Banach space of all bounded functions $x: I \rightarrow \mathbb{R}$, endowed with the supremum norm $\|x\|_{\infty}=\sup _{t \in I}|x(t)|$, and by $C(I)$ its closed subspace consisting of all the continuous functions defined on $I$.

Throughout the paper by ' $\int$,' we will denote the Lebesgue integral.

In the next two subsections, we collect basic definitions and facts which will be needed in the sequel.

\subsection{Functions of bounded variation}

Definition 1 Let $p \in[1,+\infty)$ and let $x$ be a real-valued function defined on $I$. The number

$$
\operatorname{var}_{p} x=\sup \sum_{i=1}^{n}\left|x\left(t_{i}\right)-x\left(t_{i-1}\right)\right|^{p},
$$

where the supremum is taken over all finite partitions $0=t_{0}<t_{1}<\cdots<t_{n}=1$ of $I$, is called the $p$-variation of the function $x$ over $I$.

Remark 1 If $p=1$, then the number $\operatorname{var}_{1} x$ is often referred to as the variation in the sense of Jordan of the function $x$ and usually is denoted by the symbol $\bigvee_{0}^{1} x$. If $1 \leq p \leq q$, then $\left(\operatorname{var}_{q} x\right)^{1 / q} \leq\left(\operatorname{var}_{p} x\right)^{1 / p}$ (see for example [13, Remark 2.5] or [21, p. 55]).

Definition 2 A function $\phi:[0,+\infty) \rightarrow[0,+\infty)$ is said to be a $\phi$-function, if it is continuous, unbounded, non-decreasing and such that $\phi(u)=0$ if and only if $u=0$.

Definition 3 Let $x$ be a real-valued function defined on $I$ and let $\phi$ be a given $\phi$-function. The number 


$$
\operatorname{var}_{\phi} x=\sup \sum_{i=1}^{n} \phi\left(\left|x\left(t_{i}\right)-x\left(t_{i-1}\right)\right|\right),
$$

where the supremum is taken over all finite partitions $0=t_{0}<t_{1}<\cdots<t_{n}=1$ of $I$, is called the $\phi$-variation (or variation in the sense of Young) of the function $x$ over $I$.

In the sequel, unless stated otherwise, we will assume that all the $\phi$-functions are convex.

Remark 2 It is well known that the following vector spaces of functions of bounded variation become Banach spaces, when endowed with the indicated norms:

- $B V_{p}(I)=\left\{x: I \rightarrow \mathbb{R}: \operatorname{var}_{p} x<+\infty\right\}$ with the norm $\|x\|_{B V_{p}}=|x(0)|+\left(\operatorname{var}_{p} x\right)^{1 / p}$;

- $B V_{\phi}(I)=\left\{x: I \rightarrow \mathbb{R}: x(0)=0\right.$ and $\operatorname{var}_{\phi}(\lambda x)<+\infty$ for some $\left.\lambda>0\right\}$ with the norm $\|x\|_{\phi}=\inf \left\{\lambda>0: \operatorname{var}_{\phi}(x / \lambda) \leq 1\right\}$

(see [25, Theorem 3.21]).

Remark 3 Let $\left(X,\|\cdot\|_{X}\right)$ denote $\left(B V_{p}(I),\|\cdot\|_{B V_{p}}\right)$ or $\left(B V_{\phi}(I),\|\cdot\|_{\phi}\right)$. If $x \in X$, then $x$ is a Lebesgue measurable function (see [24, Theorem 10.7 (a) and Theorem 10.9]), and furthermore, there exists a positive constant $c>0$ such that $\|x\|_{\infty} \leq c\|x\|_{X}$ for every $x \in X$. For example, if $X=B V_{1}(I)$, then $c=1$.

\subsection{Bernstein polynomials}

Let us recall that the Bernstein polynomial of order $n \in \mathbb{N}$ of a function $f \in C(I)$ is defined by the formula

$$
B_{n}(f)(t)=\sum_{k=0}^{n}\left(\begin{array}{l}
n \\
k
\end{array}\right) f\left(\frac{k}{n}\right) t^{k}(1-t)^{n-k} \quad \text { for } t \in I .
$$

If $a>0$ and $f:[-a, a] \rightarrow \mathbb{R}$, we can modify the formula (1) to obtain Bernstein polynomials of the function $f$ on the interval $[-a, a]$, which are given by the formulas

$$
B_{n}^{a}(f)(t)=\frac{1}{(2 a)^{n}} \sum_{k=0}^{n}\left(\begin{array}{l}
n \\
k
\end{array}\right) f\left(\frac{(2 k-n) a}{n}\right)(t+a)^{k}(a-t)^{n-k} \text { for } t \in[-a, a] \text { and } n \in \mathbb{N} \text {. }
$$

In the sequel, we will use the following properties of Bernstein polynomials.

Proposition 1 (cf. [19, Theorem 1.1.1]) Let $a>0$ and let $f:[-a, a] \rightarrow \mathbb{R}$ be a continuous function. Then the sequence of Bernstein polynomials $\left(B_{n}^{a}(f)\right)_{n \in \mathbb{N}}$ converges uniformly to $f$ on $[-a, a]$.

Proposition 2 (cf. [19, Section 1.8]) Let $a>0$ and let $f:[-a, a] \rightarrow \mathbb{R}$ be a continuously differentiable function. Then the sequence of derivatives of Bernstein polynomials $\left(\frac{d}{d t} B_{n}^{a}(f)\right)_{n \in \mathbb{N}}$ converges uniformly to $f^{\prime}$ on $[-a, a]$.

Proposition 3 (cf. [19, Section 2.1]) Let $a>0$ and $q \in(1,+\infty)$. If $f:[-a, a] \rightarrow \mathbb{R}$ is an absolutely continuous function such that $f^{\prime} \in L^{q}[-a, a]$, then the sequence of derivatives of Bernstein polynomials $\left(\frac{d}{d t} B_{n}^{a}(f)\right)_{n \in \mathbb{N}}$ converges to $f^{\prime}$ with respect to the $L^{q}$-norm.

Let us remark that the above properties were established in [19] for Bernstein polynomials of a function defined on the interval $I$, but they can be easily extended to Bernstein polynomials of functions defined on any interval of the form $[-a, a]$.

For thorough treatment of approximation by Bernstein polynomials, we refer the reader to, for example, $[14,19]$. 


\section{Continuity of the autonomous superposition operator}

Actually, the first result in this section is strongly connected with [9, Theorem 4.1] in which gives the necessary and sufficient condition for an autonomous superposition operator acting in the Banach space $B V_{1}(I)$ to be a mapping of higher order.

Proposition 4 Let $f: \mathbb{R} \rightarrow \mathbb{R}$ be a sum of a power series centered at 0 with the radius of convergence $\rho=+\infty$, that is, there exist real numbers $a_{0}, a_{1}, \ldots$ such that

$$
f(u)=\sum_{i=0}^{\infty} a_{i} u^{i} \quad \text { for } u \in \mathbb{R} .
$$

Then the autonomous superposition operator $F$, generated by $f$, which maps the Banach space $B V_{1}(I)$ into itself, is continuous.

Proof Let us note that the operator $F$ is well defined, since the function $f$ satisfies a local Lipschitz condition (cf. also [3, Theorem 6.13, p. 174]).

For simplicity, let us denote

$$
f_{n}(u)=\sum_{i=0}^{n} a_{i} u^{i}, \quad g_{n}(u)=\sum_{i=1}^{n} i a_{i} u^{i-1},
$$

where $u \in \mathbb{R}$ and $u^{0}=1$. Furthermore, let $x^{0}(t) \equiv 1$ and

$$
F_{n}(x)=\sum_{i=0}^{n} a_{i} x^{i} \quad \text { for every } n \in \mathbb{N} \text { and } x \in B V_{1}(I) .
$$

Since $B V_{1}(I)$ is a Banach algebra under certain norm equivalent to $\|\cdot\|_{B V_{1}}$ (cf. [3, p. 173] and [21]), the mapping $F_{n}: B V_{1}(I) \rightarrow B V_{1}(I)$ is continuous. Therefore, in order to show the continuity of $F$, it suffices to show that the sequence of mappings $\left(F_{n}\right)_{n \in \mathbb{N}}$ converges to $F$ uniformly on bounded sets. Since the function $u \mapsto\left(f_{n}-f\right)(u)$ satisfies the Lipschitz condition on every interval $[-a, a]$ with the constant $L_{n}(a)=\sup _{u \in[-a, a]}\left|f^{\prime}(u)-g_{n}(u)\right|$, we obtain

$$
\bigvee_{0}^{1}\left[F_{n}(x)-F(x)\right] \leq L_{n}(b) \bigvee_{0}^{1} x,
$$

where $b=\|x\|_{B V_{1}}$. Hence

$$
\begin{aligned}
\left\|F_{n}(x)-F(x)\right\|_{B V_{1}} & =\left|f_{n}(x(0))-f(x(0))\right|+\bigvee_{0}^{1}\left[F_{n}(x)-F(x)\right] \\
& \leq\left|f_{n}(x(0))-f(x(0))\right|+L_{n}(a) \bigvee_{0}^{1} x,
\end{aligned}
$$

which shows that $F_{n}(x) \rightarrow F(x)$ as $n \rightarrow+\infty$ uniformly on $B_{B V_{1}}(0, a)$, where $a$ is an arbitrary but fixed positive real number. This ends the proof.

The assumption of the analyticity of the generator of the autonomous superposition operator which appears in Proposition 4 can be replaced by a weaker assumption that this generator is of $C^{1}$-class. 
Theorem 2 Let $f: \mathbb{R} \rightarrow \mathbb{R}$ be a continuously differentiable function. Then the autonomous superposition operator $F: B V_{1}(I) \rightarrow B V_{1}(I)$, generated by the function $f$, is continuous.

Proof First, let us observe that the autonomous superposition operator $F$ is well defined. In order to show its continuity, similarly to the proof of Proposition 4, we will approximate $F$ by an almost uniformly convergent sequence of continuous mappings on $B V_{1}(I)$.

For a given $a>0$, let $\varphi_{a}$ denote the restriction of the function $f$ to the interval $[-a, a]$, that is, $\varphi_{a}(u)=\left.f\right|_{[-a, a]}(u)$ for $u \in[-a, a]$. Moreover, let $F_{n}: B_{B V_{1}}(0, a) \rightarrow B V_{1}(I)$ be the autonomous superposition operator generated by the $n$-th order Bernstein polynomial $B_{n}^{a}\left(\varphi_{a}\right)$ of the function $\varphi_{a}$. Since $B V_{1}(I)$ is a Banach algebra under certain norm equivalent to $\|\cdot\|_{B V_{1}}$, the operators $F_{n}$ are clearly continuous.

Now, we are going to show that the sequence $\left(F_{n}\right)_{n \in \mathbb{N}}$ converges uniformly to $F$ on $B_{B V_{1}}(0, a)$. Let us note that the function $u \mapsto\left[f-B_{n}^{a}\left(\varphi_{a}\right)\right](u)$ satisfies the Lipschitz condition on the interval $[-a, a]$ with the constant $L_{n}(a)=\sup _{u \in[-a, a]}\left|f^{\prime}(u)-\frac{\mathrm{d}}{\mathrm{du}} B_{n}^{a}\left(\varphi_{a}\right)(u)\right|$, and hence, we have

$$
\bigvee_{0}^{1}\left[F(x)-F_{n}(x)\right] \leq L_{n}(a) \bigvee_{0}^{1} x \quad \text { for } x \in B_{B V_{1}}(0, a) .
$$

Therefore, by Proposition 1 and Proposition 2, for $x \in B_{B V_{1}}(0, a)$, we get

$$
\begin{aligned}
\left\|F(x)-F_{n}(x)\right\|_{B V_{1}} & \leq\left|f(x(0))-B_{n}^{a}\left(\varphi_{a}\right)(x(0))\right|+L_{n}(a) \bigvee_{0}^{1} x \\
& \leq\left|\varphi_{a}(x(0))-B_{n}^{a}\left(\varphi_{a}\right)(x(0))\right|+a L_{n}(a) \rightarrow 0,
\end{aligned}
$$

as $n \rightarrow+\infty$, which ends the proof.

It turns out that a result similar to Theorem 2 may also be established for a wider class of generators $f: \mathbb{R} \rightarrow \mathbb{R}$, under the additional cost of weakening the topology of the target space.

Theorem 3 Suppose that a function $f: \mathbb{R} \rightarrow \mathbb{R}$ is absolutely continuous on each compact subinterval of $\mathbb{R}$. Moreover, assume that there exists a number $q \in(1,+\infty)$ such that $f^{\prime} \in$ $L^{q}[-a, a]$ for every $a>0$. Then the autonomous superposition operator $F: B V_{1}(I) \rightarrow$ $B V_{p}(I)$ is continuous, where $p$ is the conjugate number to $q$, that is, $p^{-1}+q^{-1}=1$.

Proof Fix $a>0$. First, let us observe that for arbitrary real numbers $u, w \in[-a, a]$ such that $u<w$, by the Hölder inequality, we get

$$
\begin{aligned}
|f(u)-f(w)|^{p} & \leq\left(\int_{u}^{w}\left|f^{\prime}(\tau)\right| \mathrm{d} \tau\right)^{p} \leq|u-w| \cdot\left(\int_{u}^{w}\left|f^{\prime}(\tau)\right|^{q} \mathrm{~d} \tau\right)^{\frac{p}{q}} \\
& \leq\left\|f^{\prime}\right\|_{L^{q}[-a, a]}^{p}|u-w| .
\end{aligned}
$$

Therefore

$$
\operatorname{var}_{p} F(x) \leq\left\|f^{\prime}\right\|_{L^{q}[-a, a]}^{p} \cdot \bigvee_{0}^{1} x \quad \text { for every } x \in B_{B V_{1}}(0, a),
$$

which shows that the superposition operator $F$, generated by the function $f$, is well defined.

Now, we proceed as in the proof of Theorem 2. Fix $a>0$ and denote by $\varphi_{a}$ the restriction of $f$ to the interval $[-a, a]$. Since, the Banach space $B V_{1}(I)$ is continuously embedded 
into $B V_{p}(I)$ (see Remark 1), we infer that the superposition operators $F_{n}: B_{B V_{1}}(0, a) \rightarrow$ $B V_{p}(I)$, generated by the Bernstein polynomials of the function $\varphi_{a}$, are continuous.

As before, to end the proof, it suffices to show that the sequence $\left(F_{n}\right)_{n \in \mathbb{N}}$ converges uniformly on $B_{B V_{1}}(0, a)$ to $F$. Let us observe that for every $n \in \mathbb{N}$, the function $\psi_{n}^{a}=$ $\varphi_{a}-B_{n}^{a}\left(\varphi_{a}\right)$ is absolutely continuous on $[-a, a]$ and its derivative is $q$-th power Lebesgue integrable. Hence

$$
\left|\psi_{n}^{a}(u)-\psi_{n}^{a}(w)\right|^{p} \leq\left\|\frac{\mathrm{d}}{\mathrm{du}} \psi_{n}^{a}\right\|_{L^{q}[-a, a]}^{p} \cdot|u-w| \quad \text { for } \quad u, w \in[-a, a]
$$

(cf. the formula (2)). This in turn implies that for every $x \in B_{B V_{1}}(0, a)$, we get

$$
\left\|F(x)-F_{n}(x)\right\|_{B V_{p}} \leq\left|f(x(0))-B_{n}^{a}\left(\varphi_{a}\right)(x(0))\right|+a^{\frac{1}{p}} \cdot\left\|\frac{\mathrm{d}}{\mathrm{du}} \psi_{n}^{a}\right\|_{L^{q}[-a, a]} .
$$

By Proposition 3, we infer that $\left\|\frac{\mathrm{d}}{\mathrm{du}} \psi_{n}^{a}\right\|_{L^{q}[-a, a]} \rightarrow 0$ as $n \rightarrow+\infty$, which shows that $F_{n} \rightarrow F$ uniformly on $B_{B V_{1}}(0, a)$ and ends the proof.

Since the derivative of a Lipschitz continuous function is essentially bounded (cf. [20, Theorem 7.1.5, p. 150]), from Theorem 3 we obtain the following

Corollary 1 If $f: \mathbb{R} \rightarrow \mathbb{R}$ is a function which satisfies a local Lipschitz condition, then the autonomous superposition operator $F: B V_{1}(I) \rightarrow B V_{1}(I)$, generated by $f$, is $B V_{1}-B V_{p}$ continuous for every $p>1$.

Remark 4 Let us observe that in Theorems 2 and 3, the Bernstein polynomials may be replaced by any other approximation scheme built on the polynomials approximating the given function as well as its derivative.

Remark 5 It is worth noting that the issue concerning the continuity of a superposition operator in the space $B V_{1}(I)$ was also addressed by Morse, who in [23] proved that, if a generator $f$ can be decomposed into functions of certain regularity, then the nonautonomous superposition operator, corresponding to the function $f$, acts in the space $B V_{1}(I)$ and is continuous (see [23, Theorem 7.1]). It can be easily verified that every locally Lipschitzian function exhibits the above-mentioned decomposition (just take $U\left(y_{1}, y_{2}\right)=y_{2}, A(t)=t$ and $B(x)=f(x)$ ), and therefore, Morse's result provides the complete answer to the question concerning the continuity of an autonomous superposition operator in the space $B V_{1}(I)$ raised by the authors of the monograph [2]. Unfortunately, the problem of the continuity of superposition operators in the nonautonomous case still seems far from being fully solved, since the conditions imposed on the generator $f$ in [23, Theorem 7.1] imply its continuity, whereas it is easy to see that there are nonautonomous superposition operators generated by discontinuous functions which map the space $B V_{1}(I)$ into itself continuously; it is enough, for example, to consider the function $f: I \times \mathbb{R} \rightarrow \mathbb{R}$ given by the formula

$$
f(t, x)= \begin{cases}x, & \text { if }(t, x) \in\{0\} \times \mathbb{R}, \\ 0, & \text { if }(t, x) \in(0,1] \times \mathbb{R} .\end{cases}
$$

Finally, it should also be emphasized that the proof of Morse's theorem is highly non-trivial and, although formally it is only one page long, it is based on several preceding results (which, by the way, are interesting by themselves) and therefore may be considered to be nearly 30 pages long. On the other hand, the proofs of our results, the assumptions of which are sufficient for many applications, are quite short and simple.

Now, we will pass to the more general setting. We will start with recalling some notions connected with moduli of continuity. 
Definition 4 (see [14, p. 41]) A function $\omega:[0,+\infty) \rightarrow[0,+\infty)$ is called a modulus of continuity, if it is non-decreasing, subadditive, continuous and $\omega(0)=0$.

Definition 5 (cf. [4, p. 406]) A modulus of continuity $\omega$ is said to be a modulus of continuity of a function $f:[-a, a] \rightarrow \mathbb{R}$, if $|f(t)-f(s)| \leq \omega(\delta)$ for all points $t, s$ in $[-a, a]$ such that $|t-s| \leq \delta$.

Remark 6 Every continuous function $f:[-a, a] \rightarrow \mathbb{R}$ admits a modulus of continuity, which is given by the following formula

$$
\omega_{f}(\delta)=\sup \{|f(t)-f(s)|: t, s \in[-a, a] \text { and }|t-s| \leq \delta\}, \quad \delta \geq 0
$$

(cf. [14, p. 40]). The modulus of continuity defined by (3) is often referred to as the optimal modulus of continuity of the function $f$.

In the sequel, we will need the following technical lemma, whose proof we omit, since it is similar to the proof of [14, Lemma 6.1, p. 43].

Lemma 1 Each continuous function $f:[-a, a] \rightarrow \mathbb{R}$ admits a modulus of continuity $\omega^{*}$ which is strictly increasing, unbounded, concave and such that $\omega_{f}(\delta) \leq \omega^{*}(\delta)$ for $\delta \geq 0$.

Before we proceed further, we recall the following property of modulus of continuity of Bernstein polynomials.

Proposition 5 (cf. [17]) If $f:[-a, a] \rightarrow \mathbb{R}$ is a continuous function with the optimal modulus of continuity $\omega_{f}$, then $\omega_{B_{n}^{a}(f)}(t) \leq 4 \omega_{f}(t)$ for $t \geq 0$.

Lemma 2 Let $\omega:[0,+\infty) \rightarrow[0,+\infty)$ be a concave, unbounded and strictly increasing modulus of continuity of a continuous function $f:[-a, a] \rightarrow \mathbb{R}$. Then the autonomous superposition operator $F$, generated by the function $f$, maps the closed ball $B_{B V_{1}}(0, a)$ into the subset of the space $B(I)$ consisting of functions of finite $\omega^{-1}$-variation.

Proof For an arbitrary finite partition $0=t_{0}<t_{1}<\cdots<t_{n}=1$ of the interval $I$, we have

$$
\begin{aligned}
\sum_{i=1}^{n} \omega^{-1}\left(\left|f\left(x\left(t_{i}\right)\right)-f\left(x\left(t_{i-1}\right)\right)\right|\right) & \leq \sum_{i=1}^{n} \omega^{-1}\left(\omega\left(\left|x\left(t_{i}\right)-x\left(t_{i-1}\right)\right|\right)=\sum_{i=1}^{n}\left|x\left(t_{i}\right)-x\left(t_{i-1}\right)\right|\right. \\
& \leq \bigvee_{0}^{1} x<+\infty,
\end{aligned}
$$

so $\operatorname{var}_{\omega^{-1}} F(x)<+\infty$.

At the end of this section, we are going to prove another result concerning $\phi$-variation in the proof of which we use a similar idea as in the proofs of Theorems 2 and 3 .

Theorem 4 Let $\omega:[0,+\infty) \rightarrow[0,+\infty)$ be a concave, unbounded and strictly increasing modulus of continuity of the continuous function $f:[-a, a] \rightarrow \mathbb{R}$, and let us denote by $\phi$ the function $\phi:[0,+\infty) \rightarrow[0,+\infty)$ given by the formula $\phi(s)=\left[\omega^{-1}\left(\frac{1}{5} s\right)\right]^{p}$, where $p>1$. Moreover, by $F$ and $F_{n}$, let us denote the autonomous superposition operators, generated by $f$ and $B_{n}^{a}(f)$, respectively, which map the closed ball $B_{B V_{1}}(0, a)$ into the subset of the space $B(I)$ consisting of functions of finite $\phi$-variation. Then $\operatorname{var}_{\phi}\left[F(x)-F_{n}(x)\right] \rightarrow 0$ uniformly on $B_{B V_{1}}(0, a)$. 
Proof First, we shall show that the superposition operators are well defined, that is, for every $x \in B_{B V_{1}}(0, a)$ the functions $F(x), F_{n}(x)$ are of finite $\phi$-variation. Indeed, if $0=t_{0}<\cdots<$ $t_{m}=1$ is an arbitrary finite partition of the interval $I$, then

$$
\begin{aligned}
& \sum_{i=1}^{m} \phi\left(\left|f\left(x\left(t_{i}\right)\right)-f\left(x\left(t_{i-1}\right)\right)\right|\right) \\
& \quad=\sum_{i=1}^{m}\left[\omega^{-1}\left(\frac{1}{5}\left|f\left(x\left(t_{i}\right)\right)-f\left(x\left(t_{i-1}\right)\right)\right|\right)\right]^{p} \\
& \quad \leq \sum_{i=1}^{m}\left[\omega^{-1}\left(\left|f\left(x\left(t_{i}\right)\right)-f\left(x\left(t_{i-1}\right)\right)\right|\right)\right]^{p} \leq \sum_{i=1}^{m}\left[\omega^{-1}\left(\omega\left(\left|x\left(t_{i}\right)-x\left(t_{i-1}\right)\right|\right)\right)\right]^{p} \\
& \quad=\sum_{i=1}^{m}\left|x\left(t_{i}\right)-x\left(t_{i-1}\right)\right|^{p} \leq\left(\bigvee_{0}^{1} x\right)^{p}
\end{aligned}
$$

(cf. Remark 1). This shows that $F(x)$ is of finite $\phi$-variation. To prove that $\operatorname{var}_{\phi} F_{n}(x)<+\infty$, it suffices to apply Proposition 5 along with the fact that $\omega_{f}(t) \leq \omega(t)$ for $t \geq 0$ and to follow the above reasoning.

Now, we are going to show that for a given $\varepsilon>0$ and for all but finitely many $n \in \mathbb{N}$, we have

$$
\operatorname{var}_{\phi}\left[F(x)-F_{n}(x)\right]<\varepsilon, \quad \text { whenever } \quad x \in B_{B V_{1}}(0, a) .
$$

Fix $\varepsilon>0$ and choose $\delta>0$ to be such that $\delta^{p-1}<\varepsilon / a$. Moreover, for an arbitrary finite partition $0=t_{0}<\cdots<t_{m}=1$ of the interval $I$, let us introduce the following sets

$$
N_{1}=\left\{i \in \mathbb{N}:\left|x\left(t_{i}\right)-x\left(t_{i-1}\right)\right| \leq \delta\right\} \quad \text { and } \quad N_{2}=\left\{i \in \mathbb{N}:\left|x\left(t_{i}\right)-x\left(t_{i-1}\right)\right|>\delta\right\} .
$$

If $i \in N_{1}$, then

$$
\begin{aligned}
& \left|f\left(x\left(t_{i}\right)\right)-B_{n}^{a}(f)\left(x\left(t_{i}\right)\right)-f\left(x\left(t_{i-1}\right)\right)+B_{n}^{a}(f)\left(x\left(t_{i-1}\right)\right)\right| \\
& \quad \leq\left|f\left(x\left(t_{i}\right)\right)-f\left(x\left(t_{i-1}\right)\right)\right|+\left|B_{n}^{a}(f)\left(x\left(t_{i}\right)\right)-B_{n}^{a}(f)\left(x\left(t_{i-1}\right)\right)\right| \\
& \quad \leq 5 \omega_{f}\left(\left|x\left(t_{i}\right)-x\left(t_{i-1}\right)\right|\right) \leq 5 \omega\left(\left|x\left(t_{i}\right)-x\left(t_{i-1}\right)\right|\right),
\end{aligned}
$$

and thus

$$
\begin{aligned}
\phi\left(\left|\left[F(x)-F_{n}(x)\right]\left(t_{i}\right)-\left[F(x)-F_{n}(x)\right]\left(t_{i-1}\right)\right|\right) & \leq \phi\left(5 \omega\left(\left|x\left(t_{i}\right)-x\left(t_{i-1}\right)\right|\right)\right) \\
& =\left|x\left(t_{i}\right)-x\left(t_{i-1}\right)\right|^{p}
\end{aligned}
$$

Therefore, for every $n \in \mathbb{N}$, we have

$$
\begin{aligned}
& \sum_{i \in N_{1}} \phi\left(\left|\left[F(x)-F_{n}(x)\right]\left(t_{i}\right)-\left[F(x)-F_{n}(x)\right]\left(t_{i-1}\right)\right|\right) \leq \sum_{i \in N_{1}}\left|x\left(t_{i}\right)-x\left(t_{i-1}\right)\right|^{p} \\
& =\sum_{i \in N_{1}}\left|x\left(t_{i}\right)-x\left(t_{i-1}\right)\right|^{p-1}\left|x\left(t_{i}\right)-x\left(t_{i-1}\right)\right| \leq \delta^{p-1} \sum_{i \in N_{1}}\left|x\left(t_{i}\right)-x\left(t_{i-1}\right)\right| \\
& \leq \varepsilon / a \cdot \bigvee_{0}^{1} x \leq \varepsilon .
\end{aligned}
$$

In order to estimate the sum over $N_{2}$, let us choose a number $\eta>0$ such that $\phi(2 \eta) \leq \delta \varepsilon / a$. Furthermore, assume that the inequality $\sup _{s \in[-a, a]}\left|f(s)-B_{n}^{a}(f)(s)\right| \leq \eta$ holds for all 
$n \geq n_{0}$, where $n_{0} \in \mathbb{N}$ is a certain positive integer (cf. Proposition 1). Then, for $i \in N_{2}$ and $n \geq n_{0}$, we have

$$
\begin{aligned}
& \phi\left(\left|\left[F(x)-F_{n}(x)\right]\left(t_{i}\right)-\left[F(x)-F_{n}(x)\right]\left(t_{i-1}\right)\right|\right) \\
& \quad \leq \phi\left(\left|f\left(x\left(t_{i}\right)\right)-B_{n}^{a}(f)\left(x\left(t_{i}\right)\right)\right|+\left|f\left(x\left(t_{i-1}\right)\right)-B_{n}^{a}(f)\left(x\left(t_{i-1}\right)\right)\right|\right) \\
& \quad \leq \phi(2 \eta) \leq \frac{\delta \varepsilon}{a} \leq \frac{\varepsilon}{a}\left|x\left(t_{i}\right)-x\left(t_{i-1}\right)\right| .
\end{aligned}
$$

Hence

$$
\sum_{i \in N_{2}} \phi\left(\left|\left[F(x)-F_{n}(x)\right]\left(t_{i}\right)-\left[F(x)-F_{n}(x)\right]\left(t_{i-1}\right)\right|\right) \leq \frac{\varepsilon}{a} \sum_{i \in N_{2}}\left|x\left(t_{i}\right)-x\left(t_{i-1}\right)\right| \leq \varepsilon .
$$

Hence, for all $n \geq n_{0}$, we obtain

$$
\sum_{i=1}^{m} \phi\left(\left|\left[F(x)-F_{n}(x)\right]\left(t_{i}\right)-\left[F(x)-F_{n}(x)\right]\left(t_{i-1}\right)\right|\right) \leq 2 \varepsilon,
$$

which proves that $\operatorname{var}_{\phi}\left[F(x)-F_{n}(x)\right] \rightarrow 0$ uniformly on $B_{B V_{1}}(0, a)$.

\section{Compactness results and applications}

\subsection{Hammerstein integral equation}

In this subsection, we will be interested in the problem of the existence of solutions of bounded variation in the sense of Jordan to the following nonlinear Hammerstein integral equation

$$
x(t)=\lambda \int_{0}^{1} k(t, s) f(x(s)) \mathrm{d} s, \quad t \in I,
$$

where $\lambda \in \mathbb{R}$. Let us make the following assumptions:

$1^{\circ} \quad f: \mathbb{R} \rightarrow \mathbb{R}$ is a continuous function such that:

(a) $f$ is absolutely continuous on each compact subinterval of $\mathbb{R}$;

(b) there exists a number $q \in(1,+\infty)$ such that $f^{\prime} \in L^{q}[-a, a]$ for every $a>0$;

(c) $f$ is sub-linear, that is, $\lim _{|u| \rightarrow+\infty}|f(u)| /|u|=0$;

$2^{\circ}$ the kernel $k: I \times I \rightarrow \mathbb{R}$ is such that:

(a) for every $t \in I$ the function $s \mapsto k(t, s)$ is Lebesgue measurable;

(b) the function $s \mapsto k(0, s)$ is Lebesgue integrable;

(c) $\bigvee_{0}^{1} k(\cdot, s) \leq m(s)$ for a.e. $s \in I$, where $m: I \rightarrow[0,+\infty)$ is a Lebesgue integrable function.

Remark 7 Let us recall that the condition of type $2^{\circ}$ was introduced in the paper [8]. Moreover, let us note that if the kernel $k$ satisfies the assumptions $2^{\circ}$ (a)-(c), then for every $t \in I$, the function $s \mapsto k(t, s)$ is Lebesgue integrable on $I$. Indeed, given any $t \in I$, we have

$$
|k(t, s)| \leq|k(0, s)-k(t, s)|+|k(0, s)| \leq \bigvee_{0}^{1} k(\cdot, s)+|k(0, s)| \leq m(s)+|k(0, s)|
$$

for a.e. $s \in I$,

which confirms our claim. 
Proposition 6 Let $p, r \in[1,+\infty)$ and suppose that the kernel $k: I \times I \rightarrow \mathbb{R}$ satisfies the assumptions $2^{\circ}(a)-(c)$. Then the linear integral operator $K: B V_{p}(I) \rightarrow B V_{r}(I)$, defined by the formula

$$
K x(t)=\int_{0}^{1} k(t, s) x(s) d s, \quad t \in I, x \in B V_{p}(I),
$$

is compact.

Proof For every $x \in B V_{p}(I)$, in view of the assumptions, Remarks 3 and 7, the integral

$$
\int_{0}^{1} k(t, s) x(s) \mathrm{d} s
$$

exists and is finite for every $t \in I$. Moreover, if $0=t_{0}<t_{1}<\cdots<t_{n}=1$ is an arbitrary finite partition of the interval $I$, then

$$
\begin{aligned}
\sum_{i=1}^{n}\left|K x\left(t_{i}\right)-K x\left(t_{i-1}\right)\right| & \leq \int_{0}^{1} \sum_{i=1}^{n}\left|k\left(t_{i}, s\right)-k\left(t_{i-1}, s\right)\left\|x(s) \mid \mathrm{ds} \leq \int_{0}^{1} m(s)\right\| x \|_{\infty} \mathrm{ds}\right. \\
& \leq m_{1} c_{p}\|x\|_{B V_{p}},
\end{aligned}
$$

where $m_{1}=\int_{0}^{1} m(s)$ ds and the number $c_{p}$ is such that $\|x\|_{\infty} \leq c_{p}\|x\|_{B V_{p}}$ for $x \in B V_{p}(I)$ (cf. Remark 3). Hence

$$
\left(\operatorname{var}_{r} K x\right)^{1 / r} \leq \bigvee_{0}^{1} K x \leq m_{1} c_{p}\|x\|_{B V_{p}}
$$

and therefore $\|K x\|_{B V_{r}} \leq c_{p}\left(\|k(0, \cdot)\|_{L^{1}}+m_{1}\right)\|x\|_{B V_{p}}$. This proves that the operator $K$ is well defined and continuous.

Now, we will show that $K$ is compact. If $\left(x_{n}\right)_{n \in \mathbb{N}}$ is an arbitrary sequence of elements of $B_{B V_{p}}(0,1)$, then, in view of Helly's selection theorem (see [13, Theorem 6.1;26, Theorem 2.4]), there exists a subsequence $\left(x_{n_{k}}\right)_{k \in \mathbb{N}}$, pointwise convergent to some $x \in B_{B V_{p}}(0,1)$. Let $y_{k}=x_{n_{k}}-x$ for $k \in \mathbb{N}$. We will establish that $K y_{k} \rightarrow 0$ with respect to the $B V_{r}$-norm. Given $\varepsilon>0$ let $k_{0} \in \mathbb{N}$ be such that

$$
\int_{0}^{1}|k(0, s)|\left|y_{k}(s)\right| \mathrm{d} s \leq \frac{1}{2} \varepsilon \quad \text { and } \quad \int_{0}^{1} m(s)\left|y_{k}(s)\right| \mathrm{ds} \leq \frac{1}{2} \varepsilon \quad \text { for } \quad k \geq k_{0}
$$

(note that such $k_{0}$ exists, since

$$
\int_{0}^{1}|k(0, s)|\left|y_{k}(s)\right| \mathrm{d} s \rightarrow 0 \quad \text { and } \quad \int_{0}^{1} m(s)\left|y_{k}(s)\right| \mathrm{ds} \rightarrow 0 \quad \text { as } \quad k \rightarrow+\infty
$$

by the dominated convergence theorem). If $0=t_{0}<t_{1}<\cdots<t_{m}=1$ is an arbitrary finite partition of the interval $I$, then for $k \geq k_{0}$, we have

$$
\sum_{i=1}^{m}\left|K y_{k}\left(t_{i}\right)-K y_{k}\left(t_{i-1}\right)\right| \leq \int_{0}^{1} \sum_{i=1}^{m}\left|k\left(t_{i}, s\right)-k\left(t_{i-1}, s\right)\right|\left|y_{k}(s)\right| \mathrm{ds} \leq \int_{0}^{1} m(s)\left|y_{k}(s)\right| \mathrm{ds} \leq \frac{1}{2} \varepsilon,
$$

which implies that

$$
\bigvee_{0}^{1} K y_{k} \leq \frac{1}{2} \varepsilon \quad \text { for } \quad k \geq k_{0}
$$


Thus

$$
\left\|K y_{k}\right\|_{B V_{r}} \leq\left|K y_{k}(0)\right|+\bigvee_{0}^{1} K y_{k} \leq \int_{0}^{1}|k(0, s)|\left|y_{k}(s)\right| \mathrm{ds}+\bigvee_{0}^{1} K y_{k} \leq \varepsilon \quad \text { for } k \geq k_{0},
$$

which ends the proof.

Now, we apply the above compactness result as well as the continuity result (Theorem 3) to prove the following existence theorem for Eq. (4).

Theorem 5 Let the kernel $k: I \times I \rightarrow \mathbb{R}$ and the nonlinearity $f: \mathbb{R} \rightarrow \mathbb{R}$ satisfy the assumptions $2^{\circ}(a)-(c)$ and $1^{\circ}(a)-(c)$, respectively. Then for every $\lambda \in \mathbb{R}$, there exists $a$ $B V_{1}$-solution to Eq. (4).

Proof If $\lambda=0$, then the claim is obvious. Let us note that without the loss of generality, we may assume that $\lambda=1$, and let us consider the operator $G=K \circ F: B V_{1}(I) \rightarrow B V_{1}(I)$, where the integral operator $K: B V_{p}(I) \rightarrow B V_{1}(I)$, for $p^{-1}+q^{-1}=1$, is given by (5), while the autonomous superposition operator $F: B V_{1}(I) \rightarrow B V_{p}(I)$ is generated by the function $f$. In view of Proposition 6 and Theorem 3, the operator $G$ is continuous. Let us note that it is also completely continuous since $F$ maps bounded sets of $B V_{1}(I)$ into bounded sets of $B V_{p}(I)$, which is a direct consequence of the inequality

$$
\operatorname{var}_{p} F(x) \leq\left\|f^{\prime}\right\|_{L^{q}[-a, a]}^{p} \cdot \bigvee_{0}^{1} x \quad \text { for every } \quad x \in B_{B V_{1}}(0, a)
$$

(cf. the proof of Theorem 3).

Therefore, it is enough to find a closed ball $B_{B V_{1}}(0, a) \subseteq B V_{1}(I)$ invariant under the completely continuous mapping $G$. First, let us observe that for any ball $B_{B V_{1}}(0, a)$ and $x \in B_{B V_{1}}(0, a)$ there is $\|x\|_{\infty} \leq a$ and

$$
\|F(x)\|_{\infty} \leq \sup _{s \in[-a, a]}|f(s)| .
$$

By the assumption $1^{\circ}$ (c), there exists such $R>0$ that

$$
\sup _{s \in[-R, R]}|f(s)| \cdot\left(\int_{0}^{1}|k(0, s)| \mathrm{ds}+\int_{0}^{1} m(s) \mathrm{ds}\right) \leq R .
$$

Otherwise, a sequence $\left(u_{n}\right)_{n \in \mathbb{N}}$ of real numbers would exist for which we would have

$$
\left|f\left(u_{n}\right)\right| \cdot\left(\int_{0}^{1}|k(0, s)| \mathrm{ds}+\int_{0}^{1} m(s) \mathrm{ds}\right)>n \quad \text { and } \quad\left|u_{n}\right| \leq n .
$$

The sequence $\left(u_{n}\right)_{n \in \mathbb{N}}$ may not be bounded, so there has to exist an appropriate subsequence $\left(w_{n}\right)_{n \in \mathbb{N}}$, such that $\left|w_{n}\right| \rightarrow+\infty$ and

$$
\frac{\left|f\left(w_{n}\right)\right|}{\left|w_{n}\right|} \geq\left(\int_{0}^{1}|k(0, s)| \mathrm{ds}+\int_{0}^{1} m(s) \mathrm{ds}\right)^{-1},
$$

which contradicts the assumption $1^{\circ}(\mathrm{c})$.

Therefore

$$
\|G(x)\|_{B V_{1}}=|G(x)(0)|+\bigvee_{0}^{1} G(x) \leq \int_{0}^{1}|k(0, s)||f(x(s))| \mathrm{ds}+\int_{0}^{1} m(s)|f(x(s))| \mathrm{d} s \leq R
$$


for $x \in B_{B V_{1}}(0, R)$, which implies that the ball $B_{B V_{1}}(0, R)$ is invariant under the mapping $G$. This-by the Schauder fixed-point theorem-implies that there exists a fixed point of $G$ and completes the proof.

Using the techniques developed in the paper [10], we are able to establish the following result concerning the existence of positive continuous solutions to the nonautonomous version of Eq. (4) in the class of functions of bounded variation in the sense of Jordan which satisfy a certain additional condition.

Theorem 6 Let $J \subseteq I$ be a closed set of positive Lebesgue measure. Moreover, let the functions $k: I \times I \rightarrow[0,+\infty)$ and $f: I \times[0, r] \rightarrow[0,+\infty)$, where $r>0$, be continuous. If the kernel $k$ satisfies the assumption $2^{\circ}(c)$ and if there exist positive numbers $\delta_{1}, \delta_{2}, M$ such that:

(i) $\int_{J} k(t, s) d t \geq \delta_{1}$ for each $s \in J$;

(ii) $\int_{J} k(t, s) d t \geq \delta_{2} k(u, s)$ for each $(u, s) \in I \times I$;

(iii) $f(t, u)>0$ if $M \mu(J)^{-1 / p} \leq u \leq r$ and $t \in J$, where $p>1$;

(iv) $0<M \leq r \delta_{2} \mu(J)^{-1 / q}$, where $q>1$ is such that $p^{-1}+q^{-1}=1$,

then there exits $\lambda>0$ such that the equation

$$
x(t)=\lambda \int_{0}^{1} k(t, s) f(s, x(s)) d s, \quad t \in I,
$$

has a positive ${ }^{1}$ and continuous $B V_{1}$-solution $x$ such that

$$
\left(\int_{J}[x(t)]^{p} d t\right)^{1 / p}=M .
$$

Proof The assertion follows easily from [10, Theorem 12].

Example 1 It is easy to check that the kernel $k: I \times I \rightarrow[0,+\infty)$ given by $k(t, s)=|t-s|$ satisfies the assumption $2^{\circ}$ (c) as well as the assumptions (i) and (ii) of Theorem 6 with $J=I=[0,1]$.

\subsection{Volterra-Hammerstein integral equation}

Let us consider the following nonlinear Volterra-Hammerstein integral equation

$$
x(t)=g(t)+\int_{0}^{t} k(t, s) f(s, x(s)) \mathrm{d}, \quad t \in I,
$$

where

$3^{\circ} \quad g \in C(I) \cap B V_{1}(I) ;$

$4^{\circ} \quad f: I \times \mathbb{R} \rightarrow \mathbb{R}$ satisfies the Carathéodory conditions, that is,

(a) for every $u \in \mathbb{R}$ the function $t \mapsto f(t, u)$ is Lebesgue measurable;

(b) for a.e. $t \in I$ the function $u \mapsto f(t, u)$ is continuous;

(c) $|f(t, u)| \leq m_{1}(t)$ for $(t, u) \in I \times \mathbb{R}$ with $m_{1} \in L^{p}(I)$, where $p \in(1,+\infty]$;

$5^{\circ}$ the kernel $k: \Delta \rightarrow \mathbb{R}$, where $\Delta:=\{(t, s) \in I \times I: 0 \leq s \leq t \leq 1\}$, is such that:

(a) for every $t \in I$ the function $s \mapsto k(t, s)$ is Lebesgue measurable on $[0, t]$;

\footnotetext{
${ }^{1}$ Let us recall that by a positive solution, we understand a solution which takes only nonnegative values.
} 
(b) $|k(s, s)|+\bigvee_{s}^{1} k(\cdot, s) \leq m_{2}(s)$ for a.e. $s \in I$ with $m_{2} \in L^{q}(I)$, where $q^{-1}+p^{-1}=1$;

(c) for every $\varepsilon>0$ there exists $\delta>0$ such that

$$
\int_{0}^{t}|k(\tau, s)-k(t, s)| m_{1}(s) \mathrm{d} s \leq \varepsilon,
$$

for all $(\tau, t) \in \Delta$ such that $0 \leq \tau-t \leq \delta$.

Remark 8 Let us note that if the kernel $k$ satisfies the assumptions $5^{\circ}$ (a) and (b), then for every $t \in I$ the function $s \mapsto k(t, s)$ belongs to $L^{q}[0, t]$. Indeed, given any $t \in I$, we have $|k(t, s)| \leq|k(s, s)-k(t, s)|+|k(s, s)| \leq \bigvee_{s}^{t} k(\cdot, s)+|k(s, s)| \leq m_{2}(s)$ for a.e. $s \in[0, t]$,

which confirms our claim.

Lemma 3 Let $p \in[1,+\infty]$. If the kernel $k$ satisfies the assumptions $5^{\circ}(a)$ and $(b)$, then the linear Volterra integral operator $K$ defined by

$$
K x(t)=\int_{0}^{t} k(t, s) x(s) d s, \quad t \in I,
$$

maps the space $L^{p}(I)$ into $B V_{1}(I)$ and is continuous.

Proof Let $x \in L^{p}(I)$. First, let us observe that in view of Remark 8, the integral

$$
\int_{0}^{t} k(t, s) x(s) \mathrm{d} s
$$

exists and is finite for every $t \in I$, and thus the definition of the operator $K$ does make sense.

If $0=t_{0}<\cdots<t_{n}=1$ is an arbitrary finite partition of the interval $I$, then

$$
\begin{aligned}
\sum_{i=1}^{n}\left|K x\left(t_{i}\right)-K x\left(t_{i-1}\right)\right| & =\sum_{i=1}^{n}\left|\int_{0}^{t_{i}} k\left(t_{i}, s\right) x(s) \mathrm{ds}-\int_{0}^{t_{i-1}} k\left(t_{i-1}, s\right) x(s) \mathrm{ds}\right| \\
& \leq \int_{0}^{1} \sum_{i=1}^{n}\left|\vartheta\left(t_{i}, s\right)-\vartheta\left(t_{i-1}, s\right)\right||x(s)| \mathrm{ds},
\end{aligned}
$$

where $\vartheta: I \times I \rightarrow \mathbb{R}$ is given by the following formula

$$
\vartheta(t, s)= \begin{cases}k(t, s), & \text { if }(t, s) \in \Delta, \\ 0, & \text { if }(t, s) \notin \Delta .\end{cases}
$$

Since

$$
\sum_{i=1}^{n}\left|\vartheta\left(t_{i}, s\right)-\vartheta\left(t_{i-1}, s\right)\right| \leq|k(s, s)|+\bigvee_{s}^{1} k(\cdot, s) \leq m_{2}(s) \text { for a.e. } s \in I,
$$

we infer that

$$
\sum_{i=1}^{n}\left|K x\left(t_{i}\right)-K x\left(t_{i-1}\right)\right| \leq \int_{0}^{1} m_{2}(s)|x(s)| \mathrm{ds} .
$$

This proves that $\|K x\|_{B V_{1}} \leq\left\|m_{2}\right\|_{L^{q}} \cdot\|x\|_{L^{p}}$, which means that $K$ maps the space $L^{p}(I)$ into $B V_{1}(I)$ and is continuous. 
Lemma 4 Let $p \in(1,+\infty]$. Suppose that the assumptions $5^{\circ}(a)$ and $(b)$ hold. If a bounded sequence $\left(x_{n}\right)_{n \in \mathbb{N}}$ in $L^{p}(I)$ converges almost everywhere (or in measure) to a function $x \in$ $L^{p}(I)$, then the sequence $\left(K x_{n}\right)_{n \in \mathbb{N}}$, where $K$ is given by (7), converges to $K x$ with respect to the $B V_{1}$-norm.

Proof First, let us note that $K x, K x_{n} \in B V_{1}(I)$ by Lemma 3. If $0=t_{0}<\cdots<t_{n}=1$ is an arbitrary finite partition of the interval $I$, then

$$
\begin{aligned}
& \sum_{i=1}^{n}\left|K x\left(t_{i}\right)-K x_{n}\left(t_{i}\right)-K x\left(t_{i-1}\right)+K x_{n}\left(t_{i-1}\right)\right| \\
& \quad \leq \int_{0}^{1} \sum_{i=1}^{n}\left|\vartheta\left(t_{i}, s\right)-\vartheta\left(t_{i-1}, s\right)\right|\left|x_{n}(s)-x(s)\right| \mathrm{ds},
\end{aligned}
$$

where the function $\vartheta: I \times I \rightarrow \mathbb{R}$ is defined by (8). Hence

$$
\bigvee_{0}^{1}\left[K x_{n}-K x\right] \leq \int_{0}^{1} m_{2}(s)\left|x_{n}(s)-x(s)\right| \mathrm{d} s
$$

(cf. the proof of Lemma 3), which, in view of the assumptions and Vitali's convergence theorem (see [20, Theorem 6.2.12]), shows that $\left\|K x_{n}-K x\right\|_{B V_{1}} \rightarrow 0$ as $n \rightarrow+\infty$.

The following example shows that Lemma 4 is false if $p=1$.

Example 2 Let us consider a sequence $\left(x_{n}\right)_{n \in \mathbb{N}}$ of Lebesgue integrable functions defined by the formulas

$$
x_{n}(t)=n \cdot \chi_{[0,1 / n]}(t) \quad \text { for } t \in I \text { and } n \in \mathbb{N} .
$$

Clearly, the sequence $\left(x_{n}\right)_{n \in \mathbb{N}}$ is bounded in $L^{1}(I)$ and converges almost everywhere to the zero function. If we take the kernel $k$ equal identically to 1 on $\Delta$, then

$$
K x_{n}(t)=\int_{0}^{t} x_{n}(s) \mathrm{ds}=n \cdot \min (t, 1 / n) \quad \text { for } t \in I,
$$

which shows that $\bigvee_{0}^{1} K x_{n}=1$ for $n \in \mathbb{N}$. Therefore, the sequence $\left(K x_{n}\right)_{n \in \mathbb{N}}$ cannot converge to the zero function with respect to the $B V_{1}$-norm.

Corollary 2 If the kernel $k: \Delta \rightarrow \mathbb{R}$ satisfies the assumptions $5^{\circ}(a)$ and (b) with $q=1$, then the integral operator $K: B V_{1}(I) \rightarrow B V_{1}(I)$ given by (7) is compact.

In the proof of the main result of this subsection, we will need a Vidossich-type result. Assume that $D$ is a bounded and convex subset of a normed space, and $E$ is a Banach space. Denote by $C(D, E)$ the space of all bounded and continuous functions $x: D \rightarrow E$, endowed with the supremum norm, similarly as in the case of real-valued functions.

Theorem 7 ([28, Theorem 2]) Let $F: C(D, E) \rightarrow C(D, E)$ be a continuous mapping satisfying the following conditions:

(i) the set $F(C(D, E))$ is equiuniformly continuous;

(ii) there exist $t_{0} \in D$ and $x_{0} \in E$ such that $F(x)\left(t_{0}\right)=x_{0}$ for every $x \in C(D, E)$;

(iii) for every $\varepsilon>0$ and $x, y \in C(D, E)$ the following implication holds

$$
\left.x\right|_{D_{\varepsilon}}=\left.\left.y\right|_{D_{\varepsilon}} \Rightarrow F(x)\right|_{D_{\varepsilon}}=\left.F(y)\right|_{D_{\varepsilon}},
$$

where $D_{\varepsilon}=\left\{t \in D:\left\|t-t_{0}\right\| \leq \varepsilon\right\}$; 
(iv) every sequence $\left(x_{n}\right)_{n \in \mathbb{N}}$ in $C(D, E)$ such that $\lim _{n \rightarrow \infty}\left(x_{n}-F\left(x_{n}\right)\right)=0$ has a limit point.

Then the set of fixed points of the mapping $F$ is a compact $R_{\delta}$, that is, it is homeomorphic to the intersection of a decreasing sequence of compact absolute retracts.

Let us refer the reader interested in other important results, similar to Theorem 7 , to the papers [6] and [29].

Theorem 8 If the assumptions $3^{\circ}-5^{\circ}$ hold, then the set $T$ of all continuous solutions of bounded variation in the sense of Jordan to the nonlinear Volterra-Hammerstein integral equation (6) is a compact $R_{\delta}$ in the Banach space $C(I) \cap B V_{1}(I)$ endowed with the $B V_{1}$-norm.

Proof The proof falls into two parts. First, we shall show that the mapping

$$
F(x)(t)=g(t)+\int_{0}^{t} k(t, s) f(s, x(s)) \mathrm{ds}, \quad t \in I,
$$

defined for $x \in C(I)$ satisfies the assumptions of Theorem 7; let us note that the mapping $F$ is well defined, that is, the above integral exists and is finite for every $x \in C(I)$ and $t \in I$ (cf. Remark 8).

Let $x \in C(I)$. Given $\varepsilon>0$, in view of the assumptions, there exists $\delta>0$ such that:

- $|g(t)-g(\tau)| \leq \frac{1}{3} \varepsilon$ for $t, \tau \in I$ such that $|t-\tau| \leq \delta$;

- $\int_{0}^{t}|k(\tau, s)-k(t, s)| m_{1}(s) \mathrm{d} s \leq \frac{1}{3} \varepsilon$ for $(\tau, t) \in \Delta$ such that $|t-\tau| \leq \delta$;

- $\int_{A} m_{1}(s) m_{2}(s) \mathrm{d} s \leq \frac{1}{3} \varepsilon$ for any Lebesgue measurable set $A \subseteq I$ such that $\mu(A) \leq \delta$.

Therefore, if $t, \tau \in I$ are such that $0 \leq \tau-t \leq \delta$, then

$$
\begin{aligned}
|F(x)(t)-F(x)(\tau)| \leq & |g(t)-g(\tau)|+\left|\int_{0}^{t} k(t, s) f(s, x(s)) \mathrm{ds}-\int_{0}^{\tau} k(\tau, s) f(s, x(s)) \mathrm{ds}\right| \\
\leq & |g(t)-g(\tau)|+\int_{0}^{t}|k(t, s)-k(\tau, s)||f(s, x(s))| \mathrm{ds} \\
& +\int_{t}^{\tau}|k(\tau, s)||f(s, x(s))| \mathrm{ds} \\
\leq & |g(t)-g(\tau)|+\int_{0}^{t}|k(t, s)-k(\tau, s)| m_{1}(s) \mathrm{ds}+\int_{t}^{\tau} m_{1}(s) m_{2}(s) \mathrm{ds} \\
\leq & \frac{1}{3} \varepsilon+\frac{1}{3} \varepsilon+\frac{1}{3} \varepsilon=\varepsilon .
\end{aligned}
$$

This shows that $F(x) \in C(I)$. Furthermore, let us observe that the number $\delta$ in the above reasoning is independent of $x$, which implies that the set $F(C(I))$ is equiuniformly continuous.

The continuity of the mapping $F$ is a consequence of Lemma 4 and the fact that if a sequence $\left(x_{n}\right)_{n \in \mathbb{N}}$ in $C(I)$ is uniformly convergent to $x \in C(I)$, then the sequence $\left(f\left(\cdot, x_{n}(\cdot)\right)\right)_{n \in \mathbb{N}}$, which is bounded in $L^{p}(I)$, converges a.e. to the function $t \mapsto f(t, x(t))$, $t \in I$.

The assumptions (ii) and (iii) of Theorem 7 are obviously satisfied, if we set $t_{0}=0$ and $x_{0}=g(0)$.

Hence, it suffices to prove that the mapping $F$ satisfies the Palais-Smale condition. Let $\left(x_{n}\right)_{n \in \mathbb{N}}$ be a sequence in $C(I)$ such that $\lim _{n \rightarrow \infty}\left(x_{n}-F\left(x_{n}\right)\right)=0$ with respect to the supremum norm. In view of the assumption $4^{\circ}$ and Lemma 3 , we get

$$
\|F(x)\|_{B V_{1}} \leq\|g\|_{B V_{1}}+\left\|m_{1}\right\|_{L^{p}} \cdot\left\|m_{2}\right\|_{L^{q}} \text { for } x \in C(I) .
$$


Therefore, by Helly's selection theorem there exists a subsequence $\left(F\left(x_{n_{k}}\right)\right)_{k \in \mathbb{N}}$ of $\left(F\left(x_{n}\right)\right)_{n \in \mathbb{N}}$ pointwise convergent to a function $y \in B V_{1}(I)$. Thus, $\left(x_{n_{k}}\right)_{k \in \mathbb{N}}$ is also pointwise convergent to $y$. Hence, for a.e. $t \in I$, we have $f\left(t, x_{n_{k}}(t)\right) \rightarrow f(t, y(t))$ as $k \rightarrow+\infty$, and the sequence $\left(f\left(\cdot, x_{n_{k}}(\cdot)\right)\right)_{k \in \mathbb{N}}$ is bounded in $L^{p}(I)$. This, by Lemma 4, implies that $\left(F\left(x_{n_{k}}\right)\right)_{k \in \mathbb{N}}$ converges to $F(y)=y$ with respect to the $B V_{1}$-norm, and so, since the supremum norm is weaker than the $B V_{1}$-norm, the sequence $\left(x_{n}\right)_{n \in \mathbb{N}}$ has a limit point in $C(I)$.

All the assumptions of Theorem 7 are satisfied, and therefore, the set $S$ of all continuous solutions to Eq. (6) is a compact $R_{\delta}$ in $C(I)$. To end the proof, it suffices to show that $S$ endowed with the metric $d_{\infty}$ induced by the supremum norm is homeomorphic to the set $T$ of all continuous solutions to (6) of bounded Jordan variation, endowed with the metric $d_{B V_{1}}$ induced by the $B V_{1}$-norm. Note that $S=T$ as sets (cf. the formula (9)), and since the $B V_{1}$-norm is stronger than the supremum norm, we get that the identity map id: $T \rightarrow S$ is continuous. Now, we shall show that id: $S \rightarrow T$ is also continuous. Let us take a sequence $\left(x_{n}\right)_{n \in \mathbb{N}}$ in $S$ convergent to $x_{0} \in S$. Reasoning as above, we infer that the sequence $\left(F\left(x_{n}\right)\right)_{n \in \mathbb{N}}$ converges to $F\left(x_{0}\right)$ with respect to $d_{B V_{1}}$. But $F\left(x_{n}\right)=x_{n}$ for all $n \in \mathbb{N} \cup\{0\}$, and hence $\left\|x_{n}-x_{0}\right\|_{B V_{1}} \rightarrow 0$ as $n \rightarrow+\infty$. This shows that the identity map constitutes a homeomorphism between the metric spaces $T$ and $S$ and, in consequence, it proves that the set of all continuous solutions to (6) of bounded variation in the sense of Jordan is a compact $R_{\delta}$ set in $C(I) \cap B V_{1}(I)$ with respect to the $B V_{1}$-norm.

Open Access This article is distributed under the terms of the Creative Commons Attribution 4.0 International License (http://creativecommons.org/licenses/by/4.0/), which permits unrestricted use, distribution, and reproduction in any medium, provided you give appropriate credit to the original author(s) and the source, provide a link to the Creative Commons license, and indicate if changes were made.

\section{References}

1. Ambrosio, L., Fusco, N., Pallara, D.: Functions of Bounded Variation and Free Discontinuity Problems. Oxford Mathematical Monographs, Oxford Science Publications, Clarendon Press, Oxford (2000)

2. Appell, J., Banaś, J., Merentes, N.: Bounded Variation and Around, De Gruyter Studies. In: Nonlinear Analysis and Applications, vol. 17. De Gruyter, Berlin (2014)

3. Appell, J., Zabrejko, P.P.: Nonlinear Superposition Operators. Cambridge University Press, Cambridge (1990)

4. Aronszajn, N., Panitchpakdi, P.: Extension of uniformly continuous transformations and hyperconvex metric spaces. Pac. J. Math. 6, 405-439 (1956)

5. Brauer, F.: Constant rate harvesting of populations governed by Volterra integral equations. J. Math. Anal. Appl. 56, 18-27 (1976)

6. Browder, F.D., Gupta, C.P.: Topological degree and nonlinear mappings of analytic type in Banach spaces. J. Math. Anal. Appl. 26, 730-738 (1969)

7. Bugajewska, D., Bugajewski, D., Kasprzak, P., Maćkowiak, P.: Nonautonomous superposition operators in the spaces of functions of bounded variation (submitted)

8. Bugajewski, D.: On $B V$-solutions of some nonlinear integral equations. Integral Equ. Oper. Theory 46, 387-398 (2003)

9. Bugajewski, D., Kasprzak, P.: On mappings of higher order and their applications to nonlinear equations. Commun. Pure Appl. Anal. 11(2), 627-647 (2012)

10. Bugajewski, D., Kasprzak, P.: Leggett-Williams type theorems with applications to nonlinear differential and integral equations. Nonlinear Anal. 114, 116-132 (2015)

11. Chambolle, A., Caselles, V., Cremers, D., Novaga, M., Pock, T.: An introduction to total variation for image analysis, theoretical foundations and numerical methods for sparse recovery. Radon Ser. Comput. Appl. Math. 9, 263-340. Walter de Gruyter, Berlin (2010)

12. Chan, T.F., Shen, J.: Image Processing and Analysis. Variational, PDE, Wavelet, and Stochastic Methods. Society for Industrial and Applied Mathematics (SIAM), Philadelphia (2005) 
13. Chistyakov, V.V., Galkin, O.E.: On maps of bounded $p$-variation with $p>1$. Positivity 2(1), 19-45 (1998)

14. DeVore, R.A., Lorentz, G.G.: Constructive Approximation, Grundlehren der mathematischen Wissenschaften, vol. 303. Springer, Berlin (1993)

15. Hansen, P.C., Nagy, J.G., O'Leary, D.P.: Deblurring images. Matrices, spectra, and filtering. In: Fundamentals of Algorithms, vol. 3. Society for Industrial and Applied Mathematics (SIAM), Philadelphia (2006)

16. Kim, Y., Vese, L.A.: Image recovery using functions of bounded variation and Sobolev spaces of negative differentiability. Inverse Probl. Imaging 3(1), 43-68 (2009)

17. Kratz, W., Stadtmüller, U.: On the uniform modulus of continuity of certain discrete approximation operators. J. Approx. Theory 54(3), 326-337 (1988)

18. Levin, J.J.: On a nonlinear Volterra equation. J. Math. Anal. Appl. 39, 458-476 (1972)

19. Lorentz, G.G.: Bernstein Polynomials. Chelsea Publishing Company, New York (1986)

20. Łojasiewicz, S.: An Introduction to the Theory of Real Functions. John, Chichester (1988)

21. Maligranda, L., Orlicz, W.: On some properties of functions of generalized variation. Monatsh. Math. 104(1), 53-65 (1987)

22. Mazya, V.: Sobolev Spaces: With Applications to Elliptic Partial Differential Equations, Grundlehren der mathematischen Wissenschaften, vol. 342. Springer, Heidelberg (2011)

23. Morse, A.P.: Convergence in variation and related topics. Trans. Am. Math. Soc. 41(1), 48-83 (1937)

24. Musielak, J.: Orlicz spaces and modular spaces. Lecture Notes in Mathematics, vol. 1034. Springer, Berlin (1983)

25. Musielak, J., Orlicz, W.: On generalized variations I. Studia Math. 18, 11-41 (1959)

26. Porter, J.E.: Helly's selection principle for functions of bounded p-variation. Rocky Mt. J. Math. 35(2), 675-679 (2005)

27. Rudin, L., Osher, S., Fatemi, E.: Nonlinear total variation based noise removal algorithms. Phys. D 60(1-4), 259-268 (1992)

28. Szufla, S.: On the structure of solution sets of nonlinear equations. In: Proceedings of the conference: Differential Equations and Optimal Control, Zielona Góra, pp. 33-39 (1989)

29. Vidossich, G.: On the structure of the set of solutions of nonlinear equations. J. Math. Anal. Appl. 34, 602-617 (1971)

30. Waterman, D.: On convergence of Fourier series of functions of generalized bounded variation. Studia Math. 44, 107-117 (1972) 